\title{
Efficacy and Safety of Platelet-Rich Plasma in Melasma: A Systematic Review and Meta-Analysis
}

Lingyun Zhao • Meng Hu • Qing Xiao · Runke Zhou • Yu Li •

Lidan Xiong $\cdot$ Li Li (D)

Received: April 28, 2021 / Accepted: June 26, 2021 / Published online: July 16, 2021

(C) The Author(s) 2021

\section{ABSTRACT}

Introduction: Melasma is a chronic and recurrent skin problem for which an effective therapy is currently lacking. Platelet-rich plasma (PRP) has recently emerged as a novel treatment for melasma, but to date there has been no systematic evaluation of its efficacy and safety. Methods: The Web of Science, PubMed, EMBASE, China National Knowledge Infrastructure (CNKI) and the Cochrane Library databases were searched for relevant articles using the search items "melasma," "chloasma"

Lingyun Zhao, Meng $\mathrm{Hu}$ and Qing Xiao contributed equally to this work.

Supplementary Information The online version contains supplementary material available at https:// doi.org/10.1007/s13555-021-00575-z.

L. Zhao - Q. Xiao - R. Zhou · Y. Li · L. Xiong · L. Li $(\bowtie)$

Department of Dermatology, West China Hospital,

Sichuan University, No. 37, Guo Xue Xiang,

Chengdu 610041, Sichuan, China

e-mail: hxskincos2017@qq.com

M. Hu

State Key Laboratory of Biotherapy, West China Hospital, Sichuan University, Chengdu, Sichuan, China

L. Xiong $\cdot$ L. Li

Center of Cosmetics Evaluation, West China

Hospital, Sichuan University, No. 5, Gong Xing

Road, Chengdu 610041, Sichuan, China and "platelet-rich plasma." STATA version 15.1 software was used to analyze data. Study outcomes were calculated using standardized mean differences with 95\% confidence intervals (CIs). Results: The database search identified ten studies involving 395 adult patients that met the inclusion criteria and were included in the meta-analysis. Analysis of pre- and post-treatment data from these studies revealed that the post-treatment modified Melasma Area and Severity Index (mMASI) score decreased by 1.18 (95\% CI $0.89-1.47 ; p=0.02$ ). Subjective satisfaction evaluation of PRP treatment showed that melasma treated with the combination therapy of PRP + microneedling may have been the most efficacious treatment compared to PRP alone or in combination with intradermal injection. Adverse reactions were minor, with only a few patients reporting local congestion, temporary erythema, hyperpigmentation and discoloration.

Conclusion: These results support the efficacy and safety of PRP used in combination or alone as treatment for melasma.

Keywords: Melasma; Platelet-rich plasma; Meta-analysis; Systematic review 


\section{Key Summary Points}

\section{Why carry out this study?}

Platelet-rich plasma (PRP) has recently emerged as a novel treatment for melasma.

To date, there has been no systematic evaluation of the efficacy and safety of PRP as a treatment for melasma.

\section{What was learned from the study?}

This study showed PRP is a safe and efficient treatment for melasma.

The combination of PRP and microneedle showed the more promising results for melasma treatment.

\section{INTRODUCTION}

Melasma, also known as "chloasma" or "swarthy spots", is a common pigmentation disease, mostly presenting as yellowish-brown patches on the face. It commonly occurs in middle-aged women. The etiology of melasma is complex, and related studies have shown that ultraviolet radiation, estrogen level, vascular hyperplasia and skin inflammation are inducing factors [1-3].

As melasma is stubborn and prone to relapse, a variety of treatments have been tried, but often with inconsistent results [4, 5]. Topical bleaching is a common treatment, but is often insufficient. Treatment with intense pulsed light or laser has shown conflicting results and certain side effects, such as hyperpigmentation, skin redness and scarring [6]. Previous studies have revealed that melasma is a disease that not only encompasses melanocytes, but also involves derma factors of photoaging $[7,8]$. As a result, conventional therapies which focus only on melanosomes or melanocytes may not be sufficient in removing the disease.
Platelet-rich plasma (PRP) is defined as a small volume of autologous plasma that contains a high concentration of platelets, i.e. well above normal levels, obtained by centrifugation of autologous blood and subsequent suspension of platelets [9]. PRP has been used in the treatment of alopecia, hyperpigmentation, acne and other skin diseases [10]. It is well known that the platelet alpha granules in PRP are rich in growth factors, such as platelet-derived growth factor (PDGF) and transforming growth factor (TGF)- $\beta 1$ and TGF- $\beta 2$ [11], which are associated with collagen production, wound healing and homeostasis control.

Recent studies have reported promising results of PRP in the treatment of hyperpigmentation [12-15]. However, to date there has not been a comprehensive and systematic evaluation of the therapeutic effect of PRP on melasma. We have conducted a systematic review of the literature, with the aim to study the efficacy and safety of PRP in the treatment of patients with melasma when used alone or as an adjuvant therapy and hopefully provide new clinical evidence for the treatment of melasma.

\section{METHODS}

The procedure used to conduct this systematic review and meta-analysis conforms to the PRISMA (Preferred Reporting Items for Systematic reviews and Meta-Analyses) statement [16]. This article is based on previously conducted studies and does not contain any studies with human participants or animals performed by any of the authors.

\section{Database and Search Strategy}

Two investigators independently searched the Web of Science, PubMed, EMBASE, China National Knowledge Infrastructure (CNKI) and Cochrane Library databases for original studies that had been published up to and including 23 June 2021, with no language limitation, using the main search terms of "melasma," "chloasma" and "platelet-rich plasma." The search strategy is described in detail in Electronic Supplementary Material (ESM) Table S1. 


\section{Inclusion and Exclusion Criteria}

Inclusion and exclusion criteria were established by two of the authors ( $\mathrm{LZ}$ and $\mathrm{MH}$ ) who assessed each article independently. The inclusion and exclusion criteria are described in detail in ESM Table S2.

\section{Data Extraction}

All relevant data in the included studies were independently extracted by two authors (LZ and $\mathrm{MH})$. Detailed information extracted from these studies are presented in Table 1, including the first author's name, publication year, countries/ regions, sample size, mean age of the subjects, the treatment type and follow-up period, primary outcomes and adverse reaction(s). All disputes over data were resolved through discussion.

\section{Quality Assessment}

The quality of the eight studies included in the meta-analysis was assessed using the Cochrane risk-of-bias tool (see ESM Fig. S1) in Review Manager (RevMan) Version 5.2. Most studies used controlled experimental designs, but many did not implement strict blind methods or allocation concealment. The outcome data of each study were relatively complete. In summary, the overall quality of the included studies was moderate.

\section{Statistical Analysis}

STATA version 15.1 software (StataCorp, College Station, TX, USA) was used in the data analysis. Our main outcome indicator of the efficacy of PRP for the treatment of melasma was the difference in the modified Melasma Area and Severity Index (mMASI) score before and after treatment and subjective assessment of this efficacy. We used a random-effect model to analyze the data, and heterogeneity in pooled studies was tested using the $\chi^{2}$ and $I^{2}$ tests $[17,18]$ and categorized into three levels based on the results (low heterogeneity:
$I^{2}<25 \%$, moderate heterogeneity: $I^{2}=25-75 \%$, high heterogeneity: $\left.I^{2}>75 \%\right)$. Subgroup analysis and meta-regression including six potential factors (sex, location, treatment type, publication year, age and length of follow-up) were performed to assess the presence of heterogeneity in the included studies. A sensitivity analysis was conducted to reflect the impact of individual studies on the overall results. Potential publication bias of included studies was assessed using Begg's and Egger's tests. $p$ values $<0.05$ were considered to indicate statistical significance for all analyses.

\section{RESULTS}

\section{Search Results}

A comprehensive and detailed search of the Web of Science, PubMed, CNKI, EMBASE and Cochrane Library databases identified 31 studies; this number was reduced to 16 articles after duplicate articles were removed. Following a detailed study on all the titles and abstracts of these 16 articles, we eliminated two clinical trials that had not yet completed reporting results. The remaining 14 full-text studies were evaluated for whether they met the inclusion criteria. A further four articles were excluded for the lack of essential information. Ultimately, ten studies were included in the data integration for MASI scores and subjective evaluation [19-28]. The inclusion and exclusion process of the studies is shown in Fig. 1.

\section{Study Characteristics}

The main characteristics of the included studies are presented in Table 1 . The year of publication ranged from 2018 to 2021. In terms of study design, there were four RCTs [19-22], three split-face trials $[23,24,27]$ and three self-controlled trials $[25,26,28]$. Thelocations of these trials were China (3 trials), India ( 2 trials), Egypt (3 trials), Thailand (1 trial) and Pakistan (1 trial). The studies taken together included a total of 395 patients with melasma. All of the included cases/patients were adults, $90 \%$ of whom are 


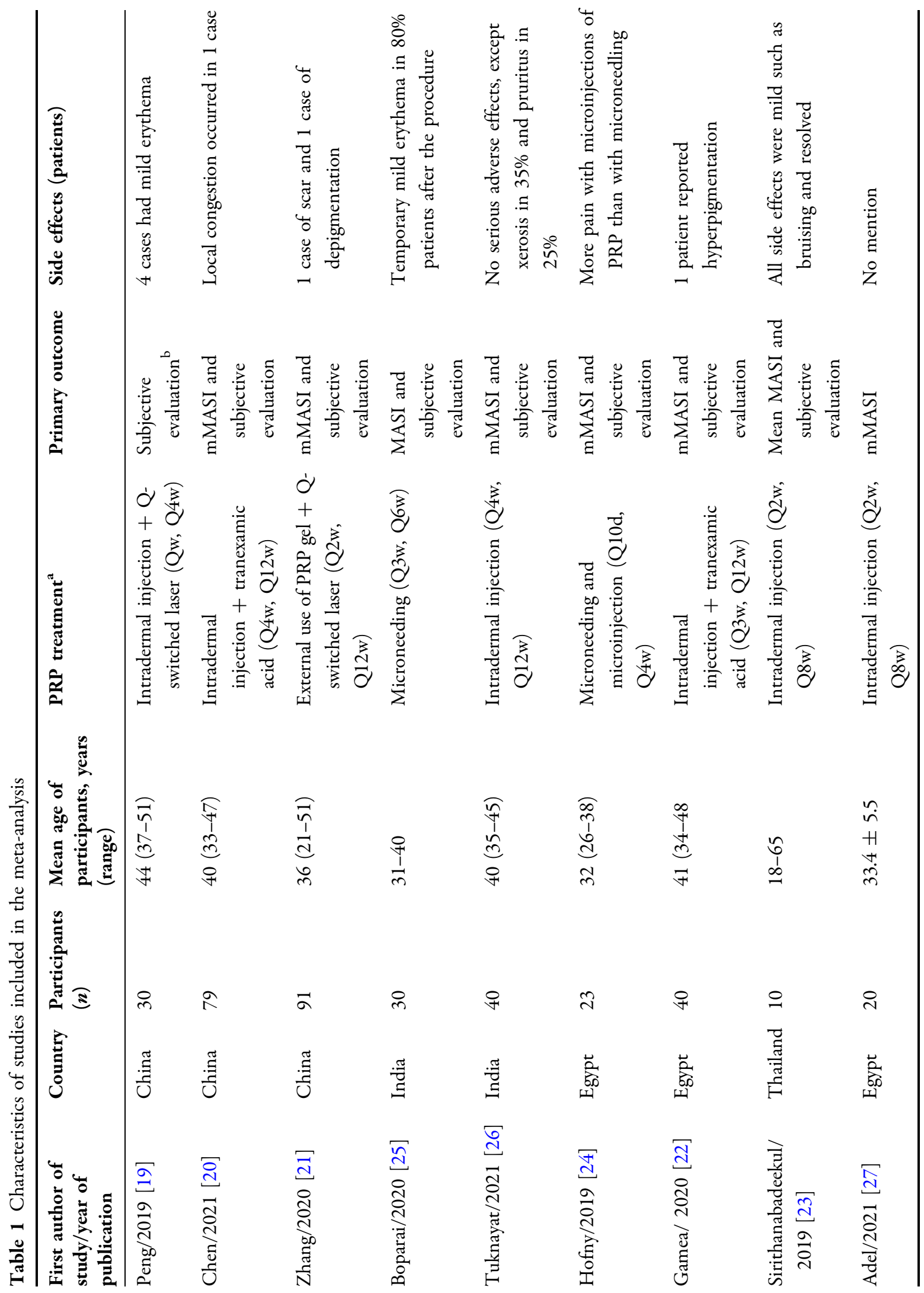




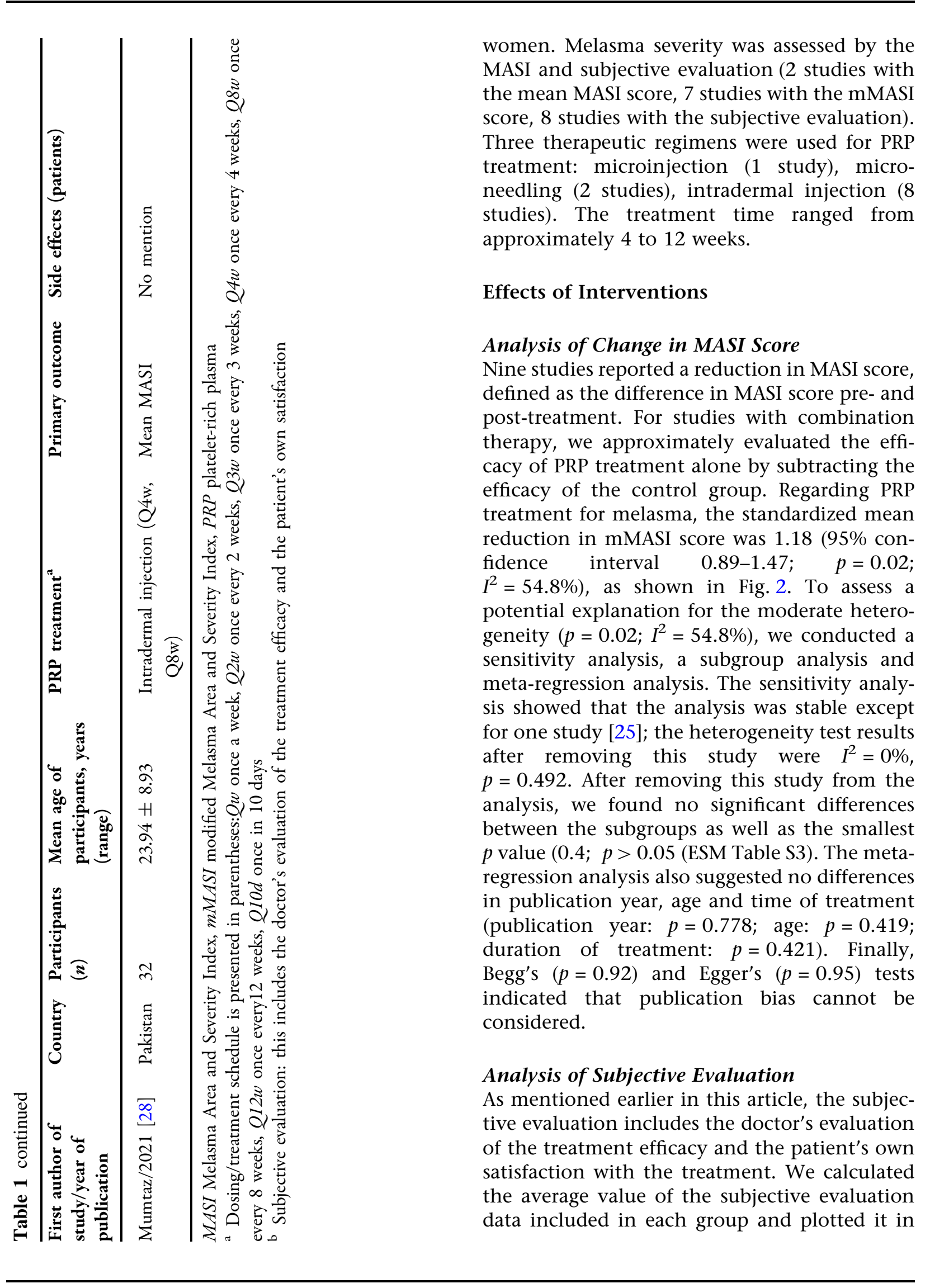




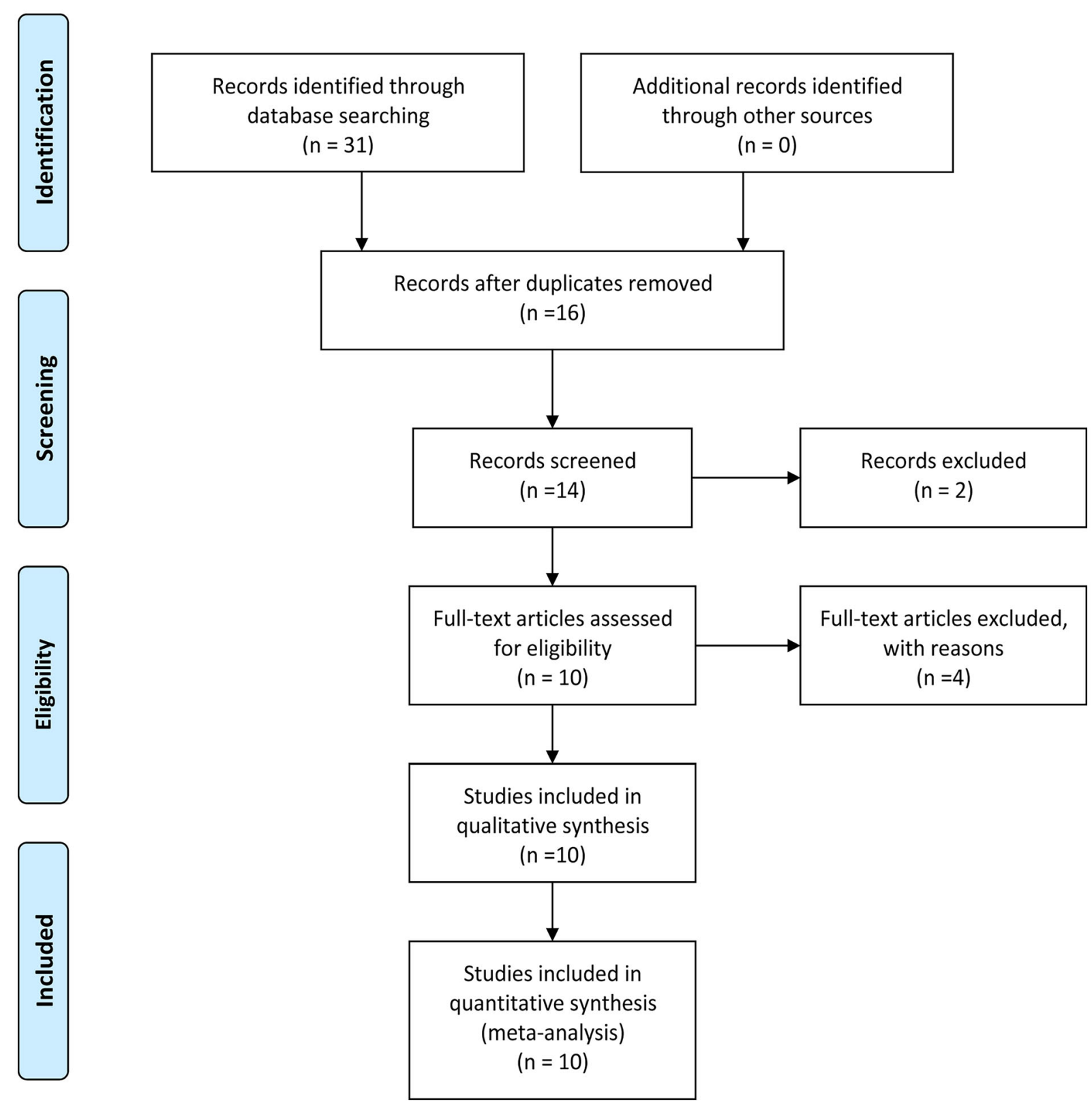

Fig. 1 PRISMA (Preferred Reporting Items for Systematic reviews and Meta-Analyses) flowchart of the selection of articles on platelet-rich plasma in the treatment of melasma

Fig. 3. Overall evaluation of the efficacy of PRP revealed that nearly one third of the participants had an excellent response and two thirds reported a high satisfaction level (including "excellent" and "satisfied"); the the rate of poor response was $9.1 \%$ (Fig. 3a). We then compared combination therapy with PRP for melasma with treatment with PRP alone; the results showed that patients showed a higher subjective satisfaction with combination therapy with PRP than for treatment with PRP alone, with the combination of PRP + Q-switched laser receiving the highest satisfaction level, at $79.5 \%$ (Fig. 3b). Finally, we compared the subjective efficacy of microneedling and intradermal injection of PRP; this analysis showed that PRP 


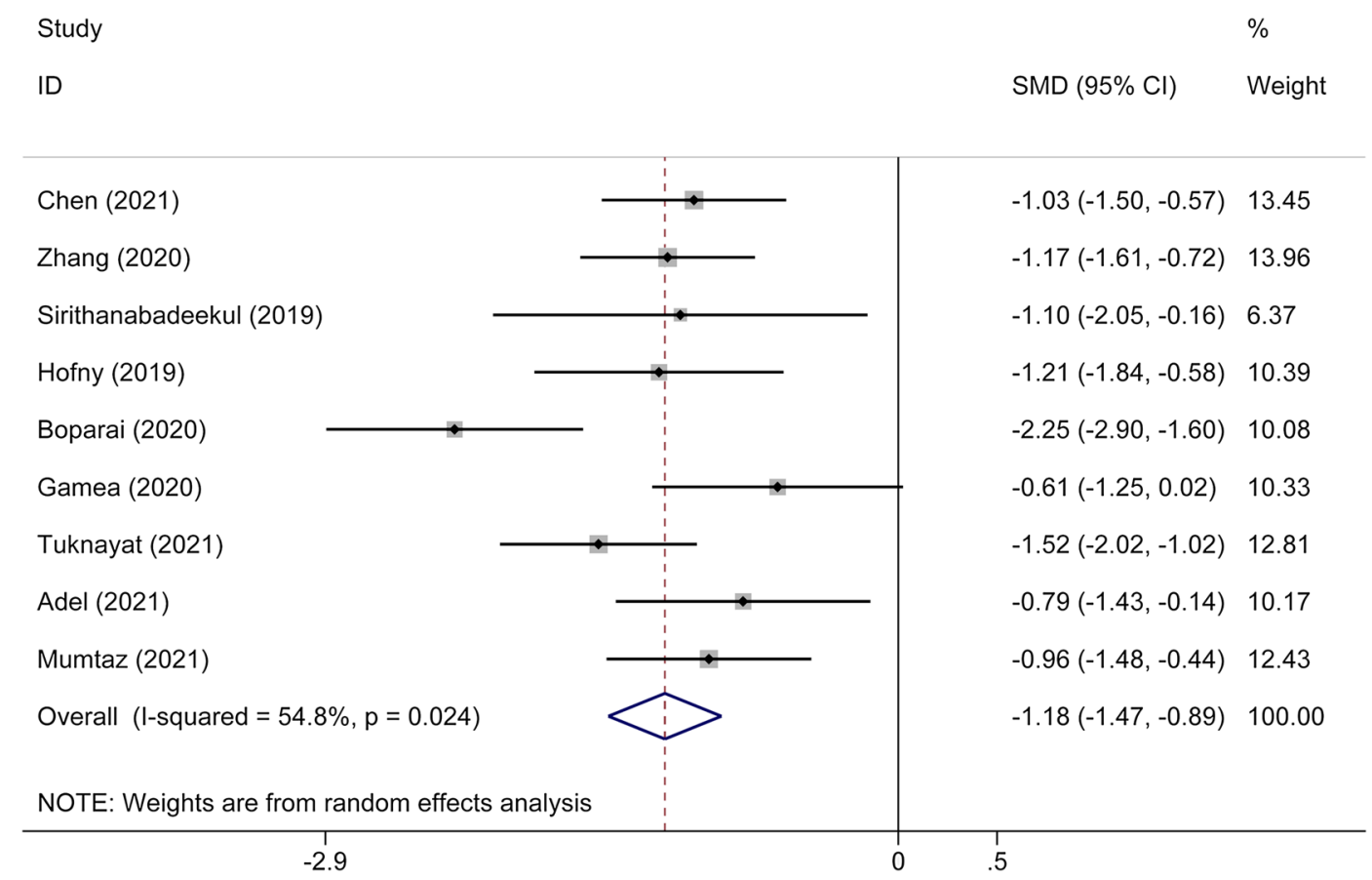

Fig. 2 Forest plot showing the comparisons of the change in Melasma Area and Severity Index (MASI) score postand pre-treatment in nine studies on treatment with

microneedling therapy received a higher satisfaction rating and had a higher excellent efficacy rate than PRP + intradermal injection (34.5 vs. $26.3 \%$ ) (Fig. 3c).

\section{Analysis of Adverse Reactions and Recurrence} No serious adverse events were reported in any of the included studies. Adverse reactions include local congestion, temporary erythema, hyperpigmentation and discoloration, all of which are few and generally mild. Observations at 3 months of follow-up showed that the average recurrence rate in the studies was about $5 \%$. Two the studies $[25,28]$ mentioned a longer period of observation, all of which showed stronger benefits and did not affect our final conclusion.

\section{DISCUSSION}

This meta-analysis of ten studies that involved 395 adults in clinical trials on PRP therapy, either in combination with other therapy or platelet-rich plasma (PRP) alone. CI Confidence interval, $S M D$ standardized mean difference

alone, showed a significant reduction in mMASI score from pre-treatment to post-treatment. The overall efficacy evaluation of PRP showed that patients or doctors had a high degree of satisfaction with the treatment of melasma by PRP. In addition, the PRP in combination with other therapies, microneedling in particular, received higher subjective satisfaction rating than PRP treatment alone and in combination with intradermal injection.

Previous studies have shown that TGF- $\beta 1$ in PRP can inhibit melanin synthesis by delaying activation of extracellular signal-regulated kinase [29]. Concomitantly, PDGF in PRP may also lead to increased skin volume (angiogenesis, collagen synthesis and extracellular matrix formation), resulting in reduced pigmentation and skin luster [14]. It has also been determined that the levels of leukocyte differentiation antigen- 4 , interleukin- 17 and cyclooxygenase- 2 are higher in patients with melasma than in normal control [30]. Thus, it can be speculated that the curative effect of PRP is not only related with pigment metabolism, but also with its 
(a)

\section{Overall evaluation of the efficacy of PRP}

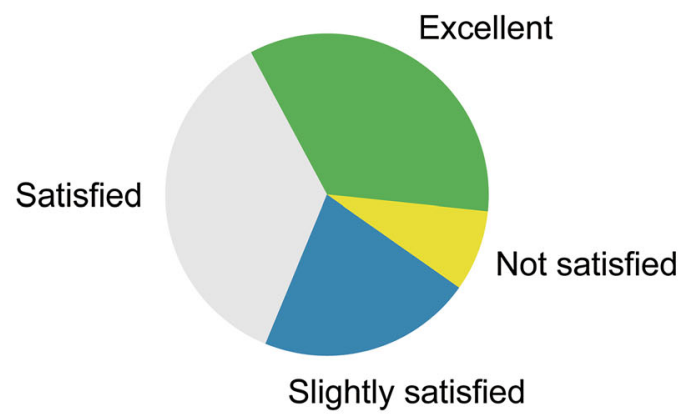

(b)

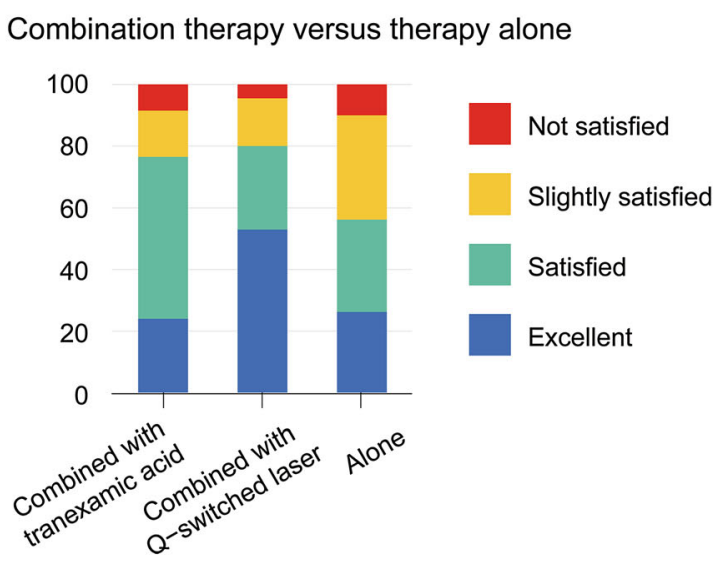

(c)

Microdeedling versus Intradermal injection

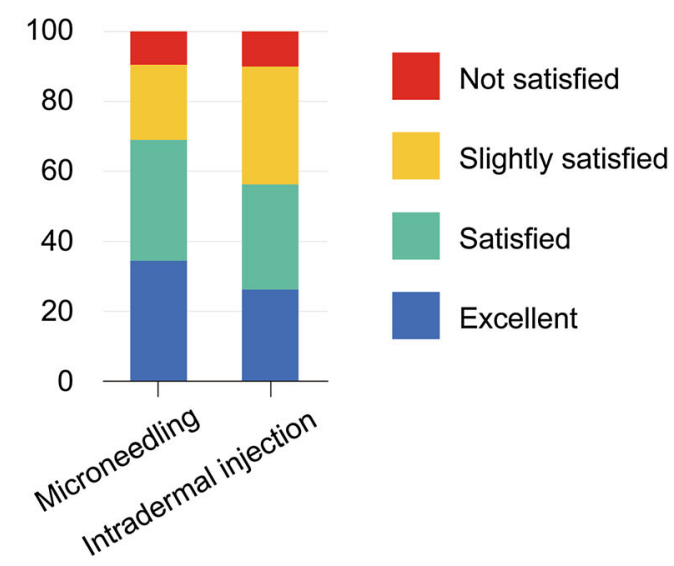

4 Fig. 3 Analysis of subjective evaluation. a Overall evaluation of the efficacy of PRP according to satisfaction level of subjects. b Difference in the proportion of subjective satisfaction ratings between PRP in combination therapy and PRP treatment alone. $\mathbf{c}$ Difference in the proportion of subjective satisfaction ratings between microneedling and intradermal injections of PRP

multiple repair function, its antibacterial or anti-inflammatory effect and its skin imperceptible blood-vessel remodeling function, all of which play a role in the several major pathology and pathogenesis of melasma, namely impaired skin barrier function, inflammation [31], pigment metabolic disorders and vascular changes.

PRP is a relatively new strategy in the treatment of melasma. To our best knowledge, this paper is the first systematic review and metaanalysis of PRP for melasma. Our research has three main advantages: First, we have comprehensively and systematically evaluated and analyzed the role of PRP in the treatment of melasma by using publication bias, regression analysis, subgroup analysis and mean subjective evaluation methods. Second, we rigorously included articles, extracted, analyzed and grouped the data by two individuals, and selected the most common and reliable MASI scoring method [32] to achieve a relatively small heterogeneity among included studies. Third, the studies we included were all very recent studies, with publication dates concentrated between 2019 and 2021.

Among the studies included in our metaanalysis, there was one study [25] with greater heterogeneity compared with the other studies that achieved a more obvious efficacy with PRP microneedle injection. Microneedles provide a minimally invasive and painless route of drug delivery by forming microchannels in the skin to enhance the penetration of active substances. It has already been used to treat skin conditions, such as wrinkles, acne scarring and discoloration, as well as to help facial rejuvenation. Combined with the results of subjective efficacy evaluation, we have reason to believe that microneedling may be a good route of PRP administration. 
In terms of improving treatment with PRP, we can try to improve or develop new administration routes of PRP to facilitate its clinical application. For example, a recent study reported that a single-hand synchronous application of PRP and microneedling by attaching a derma roller with a $5-\mathrm{ml}$ pipette secured by a sterile micropore tape [33]. Another recent study [28] showed that PRP significantly outperformed tranexamic acid in treatment for melasma from week 4 through week 24, suggesting that PRP therapy may be superior to other procedures; however, more randomized controlled studies are needed to confirm this. In addition to regaining a more balanced and stable complexion, many patients with PRP also have improved skin quality, including wrinkle levels, elasticity and skin hydration [23, 34]. Therefore, it would be valuable to offer a more personalized combination therapy to patients who want to treat melasma and improve skin quality simultaneously. In terms of mechanism study, it is meaningful to study the upstream and downstream molecules interacting with TGF- $\beta$ in melasma, such as transcription of activating protein-1, PAX3, p53, MITF, tyrosinase-related protein 1 and tyrosinase [35].

There are a number of limitations associated with this meta-analysis. First, some of the included studies were of different duration and used treatment modalities of PRP. Second, not all of the included studies were strictly randomized controlled designs, which leads to reduced credibility in their results. Third, the MASI score of patients may be affected by a number of subjectively observed differences in the grading process. Fourth, due to insufficient data in the literature, we cannot determine the influence of melasma type or severity on the therapeutic effect of PRP.

\section{CONCLUSIONS}

Our research validated that PRP is a safe and efficient treatment for melasma. Used both alone and in combination therapy, PRP treatment achieved a significant reduction of the MASI score while only incurring mild adverse reactions. Based on subjective satisfaction evaluation of PRP, combining the use of microneedling with PRP may have better efficacy in melasma treatment than PRP alone or combined with intradermal injection. As PRP in the treatment of melasma is a novel concept, in order to provide new reliable methods for the treatment of melasma, more high-quality randomized controlled clinical studies are needed to verify our conclusions.

\section{ACKNOWLEDGEMENTS}

This work was supported by 1.3.5 Project for Disciplines of Excellence of West China Hospital of Sichuan University.

Funding. This work and the journal's Rapid Service Fee was funded by 1.3.5 Project for Disciplines of Excellence of West China Hospital of Sichuan University.

Authorship. All named authors meet the International Committee of Medical Journal Editors (ICMJE) criteria for authorship for this article, take responsibility for the integrity of the work as a whole, and have given their approval for this version to be published.

Authors' Contributions. Li Li and Lingyun Zhao designed the experiment. Lingyun Zhao and Meng $\mathrm{Hu}$ extracted data. Lingyun Zhao and Meng $\mathrm{Hu}$ performed all statistical analyses. Runke Zhou and Qing Xiao participated in discussions to solve problems arising in the paper. Lidan Xiong and Li Li reviewed this article. All authors read and approved the final manuscript.

Disclosures. Lingyun Zhao, Meng $\mathrm{Hu}$, Qing Xiao, Runke Zhou, Yu Li, Lidan Xiong, Li Li have nothing to disclose.

Compliance with Ethics Guidelines. This article is based on previously conducted studies and does not contain any studies with human participants or animals performed by any of the authors. 
Conflicts of Interest. The authors declared no potential conflicts of interest with respect to the research.

Data Availability. All data generated or analyzed during this study are included in this published article/as supplementary information files.

Open Access. This article is licensed under a Creative Commons Attribution-NonCommercial 4.0 International License, which permits any non-commercial use, sharing, adaptation, distribution and reproduction in any medium or format, as long as you give appropriate credit to the original author(s) and the source, provide a link to the Creative Commons licence, and indicate if changes were made. The images or other third party material in this article are included in the article's Creative Commons licence, unless indicated otherwise in a credit line to the material. If material is not included in the article's Creative Commons licence and your intended use is not permitted by statutory regulation or exceeds the permitted use, you will need to obtain permission directly from the copyright holder. To view a copy of this licence, visit http://creativecommons.org/licenses/by$\mathrm{nc} / 4.0 /$.

\section{REFERENCES}

1. Rajanala S, Maymone MBC, Vashi NA. Melasma pathogenesis: a review of the latest research, pathological findings, and investigational therapies. Dermatol Online J. 2019;25(10):13030/ qt47b7r28c. D32510045810.

2. Kwon SH, Na JI, Choi JY, Park KC. Melasma: updates and perspectives. Exp Dermatol. 2019;28(6):704-8.

3. Sarkar R, Bansal A, Ailawadi P. Future therapies in melasma: what lies ahead? [published correction appears in Indian J Dermatol Venereol Leprol. 2020 Sep-Oct;86(5):608]. Indian J Dermatol Venereol Leprol. 2020;86(1):8-17.

4. Gupta AK, Gover MD, Nouri K, Taylor S. The treatment of melasma: a review of clinical trials. J Am Acad Dermatol. 2006;55(6):1048-65.
5. Prignano F, Ortonne JP, Buggiani G, Lotti T. Therapeutical approaches in melasma. Dermatol Clin. 2007;25(3):337-42.

6. Ho SG, Chan HH. The Asian dermatologic patient: review of common pigmentary disorders and cutaneous diseases. Am J Clin Dermatol. 2009;10(3): 153-68.

7. Kwon SH, Hwang YJ, Lee SK, Park KC. Heterogeneous pathology of melasma and its clinical implications. Int J Mol Sci. 2016;17(6):824.

8. Passeron T, Picardo M. Melasma, a photoaging disorder. Pigment Cell Melanoma Res. 2018;31(4): 461-5.

9. Wang H-L, Avila G. Platelet rich plasma: myth or reality? Eur J Dent. 2007;1:192.

10. Merchán WH, Gómez LA, Chasoy ME, AlfonsoRodríguez CA, Muñoz AL. Platelet-rich plasma, a powerful tool in dermatology. J Tissue Eng Regen Med. 2019;13(5):892-901.

11. Smyth SS, McEver RP, Weyrich AS, et al. Platelet functions beyond hemostasis. J Thromb Haemost. 2009;7(11):1759-66.

12. Kim DH, Je YJ, Kim CD, et al. Can platelet-rich plasma be used for skin rejuvenation? Evaluation of effects of platelet-rich plasma on human dermal fibroblast. Ann Dermatol. 2011;23:424-31.

13. Al-Shami SH. Treatment of periorbital hyperpigmentation using platelet-rich plasma injections. Am J Dermatol Venereol. 2014;3:87-94.

14. Cayirli M, Çalişkan E, Açikgoz G, Erbil AH, Erturk G. Regression of melasma with platelet-rich plasma treatment. Ann Dermatol. 2014;26:401-2.

15. Yew $\mathrm{CH}$, Ramasamy TS, Amini F. Response to intradermal autologous platelet rich plasma injection in refractory dermal melasma: report of two cases. J Univ Malaya Med Centre 2015;18(2):1-6.

16. Moher D, Liberati A, Etzlaff J, Altman DG, PRISMA Group. Preferred reporting items for systematic reviews and meta-analyses: the PRISMA statement. PLoS Med. 2009;6:e1000097.

17. Higgins JP, Thompson SG. Quantifying heterogeneity in a meta-analysis. Stat Med. 2002;21: 1539-58.

18. Song F, Sheldon TA, Sutton AJ, et al. Methods for exploring heterogeneity in meta-analysis. Eval Health Prof. 2001;24:126-51. 
19. Peng GK, Song JQ. Clinical efficacy of Q-modulated laser combined with PRP in the treatment of chloasma. China Med Cosmetol. 2019;9(09):60-4.

20. Chen R, Liang GX. Platelet-rich plasma combined with tranexamic acid in the treatment of chloasma. Chin J Dermatol Venerol. 201,35(2):148-151.

21. Zhang LF. Effect of platelet-rich plasma combined with Q-modulated laser therapy on chloasma. Chin J Aesthet Med. 2020;29(06):20-3.

22. Gamea MM, Kamal DA, Donia AA, Hegab DS. Comparative study between topical tranexamic acid alone versus its combination with autologous platelet rich plasma for treatment of melasma. J Dermatolog Treat. 2020. https://doi.org/10.1080/ 09546634.2020 .1781755$.

23. Sirithanabadeekul P, Dannarongchai A, Suwanchinda A. Platelet-rich plasma treatment for melasma: a pilot study. J Cosmet Dermatol. 2020;19(6): 1321-7.

24. Hofny ERM, Abdel-Motaleb AA, Ghazally A, Ahmed AM, Hussein MRA. Platelet-rich plasma is a useful therapeutic option in melasma. J Dermatolog Treat. 2019;30(4):396-401.

25. Boparai AS, Bhale G, Brar S. Evaluation of therapeutic outcome of transepidermal administration of platelet-rich plasma with microneedling in melasma. Dermatol Ther. 2020;33(6): e14358.

26. Tuknayat A, Thami GP, Bhalla M, Sandhu JK. Autologous intralesional platelet rich plasma improves melasma. Dermatol Ther. 2021;34(2): e14881. https://doi.org/10.1111/dth.14881.

27. Adel S, Serri A, Abd El-Raheem T. Study of autologous platelet-rich-plasma versus its combination with intense pulsed light in treatment of melasma. Dermatol Ther. 2021; May 26:e15008.
28. Mumtaz M, Chandio TH, Shahzad MK, Hanif N, Anwar S, Rafique S. Comparing the efficacy of patelet-rich plasma (PRP) versus tranexamic acid (4 $\mathrm{mg} / \mathrm{mL}$ ) as intradermal treatments of melasma. J Coll Phys Surg Pak. 2021;30(5):502-5.

29. Kim DS, Park SH, Park KC. Transforming growth factor-beta1 decreases melanin synthesis via delayed extracellular signal-regulated kinase activation. Int J Biochem Cell Biol. 2004;36(8): 1482-91.

30. de Medeiros ML, Araújo-Filho I, da Silva EM, et al. Effect of low-level laser therapy on angiogenesis and matrix metalloproteinase- 2 immunoexpression in wound repair. Lasers Med Sci. 2017;32(1):35-43.

31. Rodríguez-Arámbula A, Torres-Álvarez B, CortésGarcía D, Fuentes-Ahumada C, Castanedo-Cázares JP. CD4, IL-17, and COX-2 are associated with subclinical inflammation in malar melasma. Am J Dermatopathol. 2015;37(10):761-6.

32. Pandya AG, Hynan LS, Bhore R, et al. Reliability assessment and validation of the melasma area and severity index (MASI) and a new modified MASI scoring method. J Am Acad Dermatol. 2011;64(1): 78-83.e832.

33. Pathania V, Oberoi B, Shankar P, Bhatt S. Singlehanded vampire facial: combining microneedling with platelet-rich plasma for single-hand use. J Am Acad Dermatol. 2021;84(2):e77-8.

34. Shin MK, Lee JH, Lee SJ, Kim NI. Platelet-rich plasma combined with fractional laser therapy for skin rejuvenation. Dermatol Surg. 2012;38(4): 623-30.

35. Hofny ERM, Hussein MRA, Ghazally A, Ahmed AM, Abdel-Motaleb AA. Increased expression of TGF- $\beta$ protein in the lesional skins of melasma patients following treatment with platelet-rich plasma. J Cosmet Laser Ther. 2019;21(7-8):382-9. 